\title{
Diagnóstico e manejo de trauma por corpo estranho penetrante em órbita: Série de
}

\section{casos}

\author{
Diagnosis and management of trauma by penetrating foreign body in orbit: Case series \\ Diagnóstico y tratamiento del traumatismo por cuerpo extraño penetrante en órbita: Series de
}

casos

Recebido: 22/04/2021 | Revisado: 26/04/2021 | Aceito: 30/04/2021 | Publicado: 14/05/2021

\author{
Virgínia Raquel dos Santos Lucena \\ ORCID: https://orcid.org/0000-0003-3526-245X \\ Universidade Federal do Rio Grande do Norte, Brasil \\ E-mail: virlucena@hotmail.com \\ Thomas Di Nardi Medeiros \\ ORCID: https://orcid.org/0000-0001-8354-0536 \\ Universidade Federal do Rio Grande do Norte, Brasil \\ E-mail: thomasdnm@gmail.com \\ Hécio Henrique Araújo de Morais \\ ORCID: https://orcid.org/0000-0002-6450-1483 \\ Universidade Federal do Rio Grande do Norte, Brasil \\ E-mail: heciomorais@hotmail.com \\ Erasmo Freitas de Souza Júnior \\ ORCID: https://orcid.org/0000-0003-1714-9226 \\ Universidade Estadual do Rio Grande do Norte, Brasil \\ E-mail: erasmo_jn@hotmail.com \\ André Vajgel Fernandes \\ ORCID: https://orcid.org/0000-0003-1534-7595 \\ Universidade de Pernambuco, Brasil \\ E-mail: avajgel@hotmail.com
}

\begin{abstract}
Resumo
Introdução: Corpos estranhos intraoculares são a maior causa de lesões traumáticas em órbita, possuem características diversas, que abrangem do edema palpebral ao trauma órbito craniano, resultando em sequelas graves e algumas vezes fatais. Objetivo: O objetivo do presente estudo é apresentar uma série de casos de trauma por corpo estranho penetrante na órbita ocular associado a uma revisão da literatura sobre o tema. Desenho: Estudo retrospectivo de casos. Metodologia: Revisão de acordo com a declaração PRISMA, por meio das bases de dados PubMed, Science Direct e SciELO, além dos relatos de casos e análise retrospectiva de 12 pacientes submetidos à diferentes manejos. Resultados: A idade média dos pacientes foi de 31,16 anos, sendo 91,6\% dos pacientes do sexo masculino, a etiologia em $50 \%$ dos casos foi por material de madeira, em $41,7 \%$ foi por metal e em $8,3 \%$ por vidro. A tomografia computadorizada foi utilizada em todos os casos fornecendo resultados altamente efetivos. Em 58,3\% dos casos ocorreram na órbita esquerda. Cinquenta por cento dos casos tratados evoluíram sem sequelas. Conclusão: $\mathrm{O}$ diagnóstico precoce e manejo imediato de lesões orbitárias são imprescindíveis para um prognóstico positivo, assim como exames de imagem adequados. A tomografia computadorizada, a ressonância magnética e a angiografia por tomografia computadorizada são modalidades de imagem frequentemente utilizadas e eficazes.
\end{abstract}

Palavras-chave: Órbita; Corpos estranhos; Traumatismos; Olho.

\begin{abstract}
Introduction: Intraocular foreign bodies are the major cause of traumatic injuries in orbit, they have different characteristics, ranging from eyelid edema to cranial orbit trauma, they occur in severe and sometimes fatal sequelae. Objective: The objective of the present study is to present a series of cases of trauma by a penetrating foreign body in the eye socket associated with a literature review on the subject. Design: Retrospective case study. Methodology: Review according to the PRISMA statement, using the PubMed, Science Direct and SciELO databases, in addition to the case reports and retrospective analysis of 12 patients on different managements. Results: The mean age of the patients was 31.16 years, with $91.6 \%$ of the patients being male, the etiology in $50 \%$ of the cases of the wood material, in $41.7 \%$ it was by metal and in $8,3 \%$ of glass. Computed tomography was used in all cases obtained with effective results. In $58.3 \%$ of the cases, they occurred in the left orbit. Fifty percent of treated cases evolved without sequelae. Conclusion: Early diagnosis and immediate management of orbital lesions are essential for a positive prognosis, as well as adequate imaging tests. Computed tomography, magnetic resonance and computed tomography angiography are frequently used and effective imaging modalities.
\end{abstract}

Keywords: Orbit; Foreign bodies; Traumatism; Eye. 


\begin{abstract}
Resumen
Introducción: Los cuerpos extraños intraoculares son la principal causa de lesiones traumáticas en órbita, tienen diferentes características, que van desde el edema palpebral hasta el traumatismo de la órbita craneal, ocurren en secuelas severas y en ocasiones fatales. Objetivo: El objetivo del presente estudio es presentar una serie de casos de trauma por cuerpo extraño penetrante en la cuenca del ojo asociado a una revisión de la literatura sobre el tema. Diseño: estudio de caso retrospectivo. Metodología: Revisión según declaración PRISMA, utilizando las bases de datos PubMed, Science Direct y SciELO, además de los reportes de casos y análisis retrospectivo de 12 pacientes en diferentes manejos. Resultados: A idade média dos pacientes foi de 31,16 anos, sendo $91,6 \%$ dos pacientes do sexo masculino, a etiologia em $50 \%$ dos casos do material da madeira, em $41,7 \%$ foi por metal e em $8,3 \%$ de vidrio. En todos los casos se utilizó tomografía computarizada obtenida con resultados efectivos. En el $58,3 \%$ de los casos ocurrieron en la órbita izquierda. El cincuenta por ciento de los casos tratados evolucionó sin secuelas. Conclusión: El diagnóstico precoz y el manejo inmediato de las lesiones orbitarias son fundamentales para un pronóstico positivo, así como las pruebas de imagen adecuadas. La tomografía computarizada, la resonancia magnética y la angiografía por tomografía computarizada se utilizan con frecuencia como modalidades de imagen eficaces.
\end{abstract}

Palabras clave: Orbita; Cuerpos extraños; Trauma; Ojo.

\title{
1. Introdução
}

Corpos estranhos intraoculares são a maior causa de lesões traumáticas em órbita. Possuem características diversas, que abrangem do edema palpebral, ao trauma órbito craniano, resultando em sequelas graves e algumas vezes fatais. A coleta da história clínica, exame físico completo e exames de imagem adequados são imprescindíveis para determinar o trajeto do objeto, estruturas anatômicas lesadas, extensão das lesões, e possíveis comprometimentos neurovasculares (Cho et al., 2017; Mzimbiri et al., 2016).

Os corpos estranhos intra-orbitais são comumente divididos em duas categorias: inorgânico e orgânico. Os inorgânicos são metálicos ou não metálicos (vidro, plástico, espuma, dentre outros) e os orgânicos normalmente encontrados, incluem: madeira, solo, matéria vegetal e outras. $\mathrm{O}$ exame de imagem adequado e a história clínica bem documentada sugerem uma maior margem de sucesso para lesões intraorbitais sutis (Joos et al, 2016).

A maioria dos corpos estranhos intraoculares é metálica, vidro e madeira orgânica. A energia cinética do corpo estranho dissipada nos tecidos é um dos fatores determinantes para a caracterização da lesão. Em baixa velocidade, o objeto resulta em ruptura e laceração direta dos tecidos. Em alta velocidade pode provocar cavitação do tecido atingido e ondas de choque diretamente relacionadas ao dano cerebral e prognóstico desfavorável (Chowdhury et al., 2016). Além disso, as características do objeto e ângulo de aproximação são relevantes quanto à capacidade de penetração do objeto (Baranwal et al., 2016).

Corpos estranhos na cavidade orbitária possuem diversas classificações. Shukla et al., em 2016, dissertaram acerca de uma nova classificação de corpos estranhos oculares, reconhecendo os três tipos principais: oculares, anexos e mistos. Os oculares podem ser intraoculares (atingindo íris, lentes, coroide, retina, dentre outros), extraoculares (na superfície da córnea ou na esclera) e intramurais. Os anexos podem atingir pálpebras, órbita, ductos lacrimais e conjuntiva. Os mistos ocorrem quando múltiplas estruturas extraoculares ou intraoculares coexistem.

Um sistema para classificação mecânica das lesões do olho é utilizado na caracterização dos traumas oculares. O sistema classifica as lesões em trauma ocular aberto e fechado. No trauma ocular aberto, pode haver laceração ou ruptura. A laceração pode ser ainda perfurante, penetrante ou por corpo estranho intraocular. O trauma ocular fechado é dividido em contuso, lamelar ou por corpo estranho superficial.

Em virtude da anatomia da órbita, a região possui grande relação com estruturas neurovasculares importantes. Corpos estranhos intraorbitais que atravessam o teto frontal, fino, podem atingir o canal óptico e a fissura orbital superior, os quais concedem acesso ao conteúdo intracraniano (Albert et al., 1985; Balasubramanian et al., 2009; Del Verme et al., 2020). Existe alta probabilidade de lesão intracraniana nos traumas oculares abertos por corpo estranho intraocular. O teto orbital é a via mais comum devido à fragilidade da placa orbital superior do osso frontal no assoalho anterior da fossa craniana. Pela fissura 
orbital superior, os corpos estranhos podem atingir o tronco cerebral através do seio cavernoso (Gönül et al., 2005). Além disso, com o atraso do diagnóstico, pode ocorrer celulite orbital, infecção de sistema nervoso central, fístula do líquido cefalorraquidiano, aneurisma traumático e hemorragia intracraniana (Park et al., 2018).

O diagnóstico precoce das lesões orbitárias torna-se imprescindível para definição do manejo adequado. As radiografias simples são notoriamente não confiáveis para detectar perturbações orbitais, podendo ser úteis para a identificação de fraturas e corpos estranhos radiopacos (Okay et al., 2009). A tomografia computadorizada multislice deve ser considerada obrigatória para avaliação completa (Kane et al., 1991).

Corpos estranhos intra-orbitais metálicos possuem bom prognóstico oftalmológico quando seu caminho não atravessa o globo e a integridade do globo é mantida. Possuem prognóstico oftalmológico duvidoso em traumas penetrantes. A tomografia computadorizada auxilia na localização adequada do corpo estranho metálico, na estimativa de sua consistência, tamanho, trajetória, e na relação com o tecido orbital adjacente, além de ajudar no planejamento cirúrgico. No entanto, os artefatos de listras devem ser diferenciados de qualquer protrusão do corpo estranho metálico linear (Hada et al., 2016).

Em função do baixo custo e da facilidade de execução, a radiografia convencional é, às vezes, o exame de primeira escolha em objetos metálicos e de vidro. A literatura explica que o vidro é radiopaco nas radiografias, desde que os fragmentos sejam maiores $0,5 \mathrm{~mm}$ e não estejam projetados sobre osso (Srivastava et al., 2012). Os fragmentos de vidro podem se romper dentro da ferida, e tais lesões devem ser minuciosamente investigadas, incluindo a investigação de lesões vasculares, complementado com angiotomografia e tomografia computadorizada (Assoumane et al., 2019).

Os achados da tomografia computadorizada (TC) podem ser mal interpretados como pneumoencéfalo e pneumoórbita. Em casos de perfuração por madeira, a aparência da madeira é semelhante à do tecido mole, dificultando a detecção (Wilson et al., 2016; Di Gaeta et al., 2017). Embora a ressonância magnética seja mais sensível e específica, uma angiografia por TC com reconstrução tridimensional pode fornecer parâmetros confiáveis sobre o modo de intervenção necessário (Balasubramanian et al., 2009).

Quanto ao tratamento de lesões orbitárias, é importante ressaltar que procedimentos cirúrgicos para remoção de corpos estranhos orbitais não estão isentos de riscos, incluem: falha na localização do corpo estranho, fragmentação durante a remoção, lesões inadvertidas nas estruturas orbitais (incluindo o nervo óptico e a musculatura extrínseca do olho). A suspeita clínica ou a confirmação imaginológica devem levar à exploração cirúrgica, com a maioria dos pacientes obtendo melhora visual após a remoção do corpo estranho (Nasr et al., 1999). A intervenção cirúrgica é indicada comumente em traumas com presença de corpo estranho agudo, sinais de infecção, proptose, motilidade restrita, massa orbital palpável, compressão do nervo óptico, abscesso, suspeita de material orgânico e formação de fístula (Fulcher et al., 2002).

Nesse contexto, o presente estudo abordará uma visão retrospectiva de diferentes casos de corpos estranhos em cavidade orbitária, quanto ao diagnóstico e manejo, associada a uma revisão de literatura.

\section{Metodologia}

\subsection{Série de Casos}

O presente estudo analisou os casos de doze pacientes atendidos em unidade de emergência com corpos estranhos impactados na região orbital, entre os anos de 2015 a 2019. Todos os pacientes foram submetidos a cirurgias com diferentes abordagens, dependendo do grau de comprometimento morfofuncional, sendo encaminhados ao centro cirúrgicos. Também foram analisados os respectivos tratamentos profiláticos e a terapia antibiótica prescrita. Os dados elencados de cada caso específico foram etiologia da lesão, tipo de corpo estranho, sinais e sintomas, exame de imagem utilizado, tratamento e desfecho. Foram obtidos os termos de Consentimento Livre e Esclarecido (TCLE) de todos os pacientes descritos no presente estudo. 


\subsection{Revisão de Literatura}

Foi realizada busca ativa nas plataformas de bases de dados PubMed, Science Direct e Scielo, com o objetivo de encontrar relatos de casos, séries de casos e estudos observacionais. Foram utilizados nas buscas os descritores "Foreign body and Orbitocranial", "Orbital and Foreign body" e "Orbital cavity and injury", possibilitando a análise dos resumos e títulos dos trabalhos. Após a leitura completa de todos os artigos, 16 foram descartados dos 51 selecionados pelos títulos e resumos, por não abrangerem, de acordo com os critérios de inclusão, o tema selecionado, resultando na seleção de 35 trabalhos (Figura 1).

Figura 1 - Representação esquemática dos métodos de identificação, triagem, elegibilidade e inclusão de trabalhos, adaptada de acordo com o PRISMA Flow Diagram.

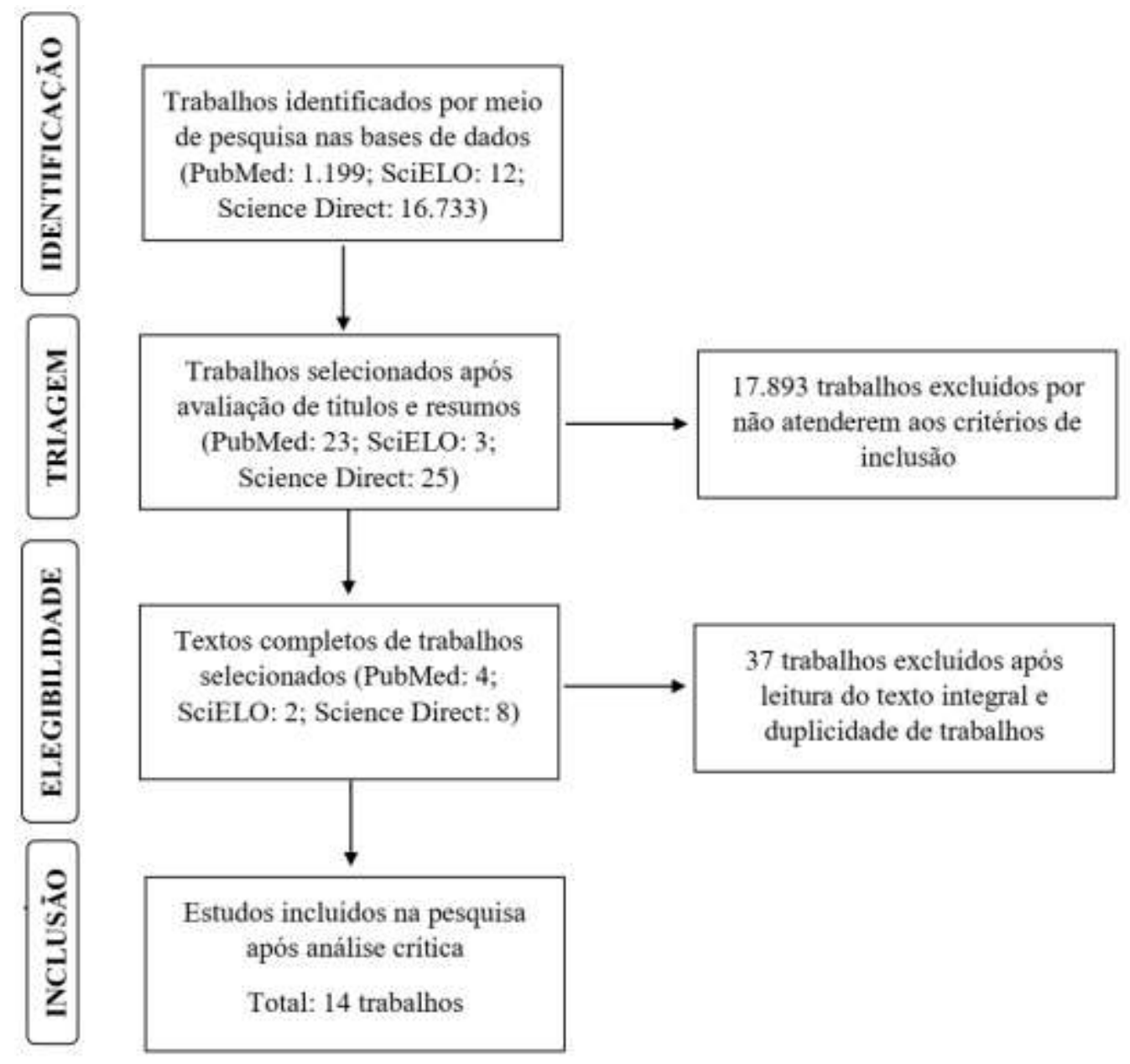

Fonte: Autores.

Desse modo, foi possível o levantamento e análise da literatura, almejando-se uma análise comparativa dos manejos e tratamentos realizados nos casos relatados.

\section{Resultados e Discussão}

\subsection{Descrição dos casos}

A Tabela 1 reúne os casos observados e manejados no presente estudo. Em 5 dos 10 casos, as lesões foram por material de madeira. 
Tabela 1 - Manejos de corpos estranhos em órbita nos casos descritos.

\begin{tabular}{cccccccc}
\hline Caso & Sexo & Idade & Corpo estranho & Localização & $\begin{array}{c}\text { Exame de } \\
\text { imagem }\end{array}$ & Tratamento & Desfecho \\
\hline $\mathbf{1}$ & Masculino & 32 & Culatra de arma de fogo & Órbita direita & TC & Remoção cirúrgica & Amaurose \\
\hline $\mathbf{2}$ & Masculino & 12 & Madeira & Órbita direita & TC & Remoção cirúrgica & Sem sequelas \\
\hline $\mathbf{3}$ & Masculino & 58 & Metal & Órbita esquerda & TC & Remoção cirúrgica & Perda da visão \\
\hline $\mathbf{4}$ & Masculino & 40 & Madeira & Órbita direita & TC & Remoção cirúrgica & Sem sequelas \\
\hline $\mathbf{5}$ & Masculino & 37 & Madeira & Órbita direita & TC & Remoção cirúrgica & Sem sequelas \\
\hline $\mathbf{6}$ & Masculino & 22 & Madeira & Órbita esquerda & TC & Remoção cirúrgica & Sem sequelas \\
\hline $\mathbf{7}$ & Masculino & 25 & Madeira & Órbita esquerda & TC & Remoção cirúrgica & Sem sequelas \\
\hline $\mathbf{8}$ & Masculino & 14 & Projétil de arma de fogo & Órbita direita & TC & Remoção cirúrgica & Perda da visão \\
\hline $\mathbf{9}$ & Masculino & 32 & Prego & Órbita esquerda & TC & Remoção cirúrgica & Perda da visão \\
\hline $\mathbf{1 0}$ & Feminino & 45 & Vidro & Órbita esquerda & TC & Remoção cirúrgica & Sem sequelas \\
\hline $\mathbf{1 1}$ & Masculino & 24 & Madeira & Órbita esquerda & TC & Remoção cirúrgica & Perda da visão \\
\hline $\mathbf{1 2}$ & Masculino & 33 & Culatra de arma de fogo & Órbita esquerda & TC & Remoção cirúrgica & Perda da visão \\
\hline
\end{tabular}

Fonte: Autores.

Nos casos 2, 4, 5, 6, 7 e 11 (Figuras 2A-D), as lesões causaram dor, amaurose e oftalmoplegia evidenciada sob teste de mobilidade ocular. Equimose periórbitária estava presente nos pacientes avaliados. Nos casos 2, 4, 6 e 7 ao avaliar-se a TC, foi possível observar a trajetória do corpo estranho, material hipodenso o qual não impactou globo ou estrutura neurovascular importante. Após procedimento cirúrgico de retirada do objeto, evoluíram também sem sequelas. No caso 5, ao se utilizar a TC, foi possível observar trauma direto do corpo estranho ao globo ocular. Os cinco pacientes com trauma por madeira não apresentaram sequelas. $\mathrm{O}$ caso 11 foi o único que evoluiu com cegueira. 
Figura 2 - A) Vítima de agressão com corpo estranho de madeira empalado em cavidade orbitária, apresentando tomografia computadorizada, evidenciando hipodenso transfixando região orbitária esquerda em visão axial e material de $8 \mathrm{~cm}$ retirado (caso 11). B) Corpo de madeira alojado em cavidade orbital (caso 4). C) Inserção de corpo estranho de madeira em órbita direita após acidente em área rural (caso 2). D) Inserção de corpo estranho de madeira em órbita direita 5 dias após o acidente, evidenciando edema da região periorbital e quemose conjuntival (caso 5).

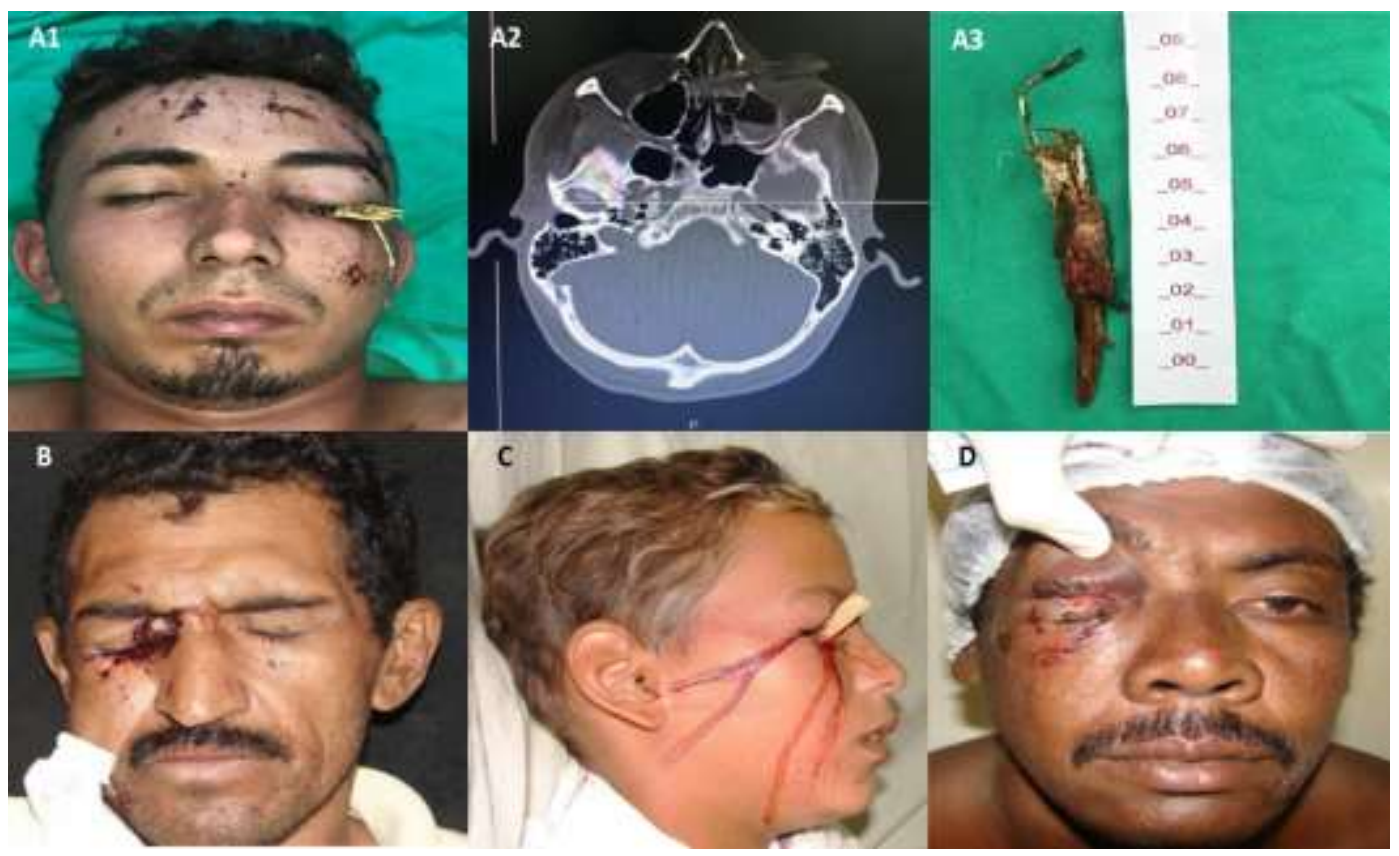

Fonte: Autores.

Os pacientes 1, 3, 8, 9 e 12 apresentaram lesão por metal (Figuras 3A-C). Todos evoluíram com cegueira. O primeiro paciente teve lesão penetrante de órbita, por culatra de rifle, com inserção na região infraorbitária direita, admitido dentro do protocolo ATLS, avaliado com tomografia computadorizada (TC) com reconstrução 3D, revelando múltiplas fraturas, de osso zigomático, esfenóide e maxila, com corpo estranho alojado em fossa infratemporal. O objeto metálico era palpável na região temporal. Paciente apresentou queixa de amaurose e dor no olho direito. Exame oftalmológico usando biomicroscopia demonstrou midríase paralítica, córnea intacta, hifema e quemose conjuntival com hemorragia. $\mathrm{O}$ exame fundoscópico revelou hemorragia vítrea inferior, hemorragia retiniana, descolamento da coroide, descolamento vítreo e papilar. O corpo estranho foi removido com acesso temporal. O segundo paciente apresentou lesão orbitária por serra, com suspeita inicial de dano neurológico (lesão de meninge). A radiografia convencional não foi suficiente para confirmar a trajetória do corpo estranho e identificar sua localização. Ao ser submetido à TC, foi possível observar a inexistência de lesão neurológica, lesão em teto orbital esquerdo e osso etmóide. O paciente foi conduzido ao bloco cirúrgico, sob anestesia geral, para retirada cirúrgica do objeto pela própria ferida causada por ele. $\mathrm{O}$ terceiro paciente foi admitido com penetração de cavidade orbitária esquerda por fragmento serra metálica. Foi encaminhado ao exame de tomografia computadorizada para estudo pré-operatório, e melhor visualização do projétil, que apresentava aspecto hiperdenso, assim como no caso 9. O quinto paciente (caso 12) também apresentou lesão penetrante culatra, sendo avaliada por tomografia computadorizada (TC) com reconstrução 3D, apresentou queixa de amaurose e dor no olho esquerdo. O exame oftalmológico usando biomicroscopia demonstrou midríase paralítica, hifema e quemose conjuntival com hemorragia. O exame fundoscópico revelou hemorragia vítrea inferior, hemorragia retiniana, descolamento da coroide, descolamento vítreo e papilar. 
Figura 3 - A) Inserção de culatra de arma de fogo em órbita direita após acidente doméstico (caso 1). B) Vítima de acidente de trabalho, apresentando síndrome de Jael, com inserção de fragmento de serra metálica em órbita esquerda (caso 3). C) Inserção de culatra de culatra de arma de fogo em órbita esquerda após acidente doméstico e tomografia computadorizada em corte axial, evidenciando material hiperdenso em órbita esquerda (caso 12).

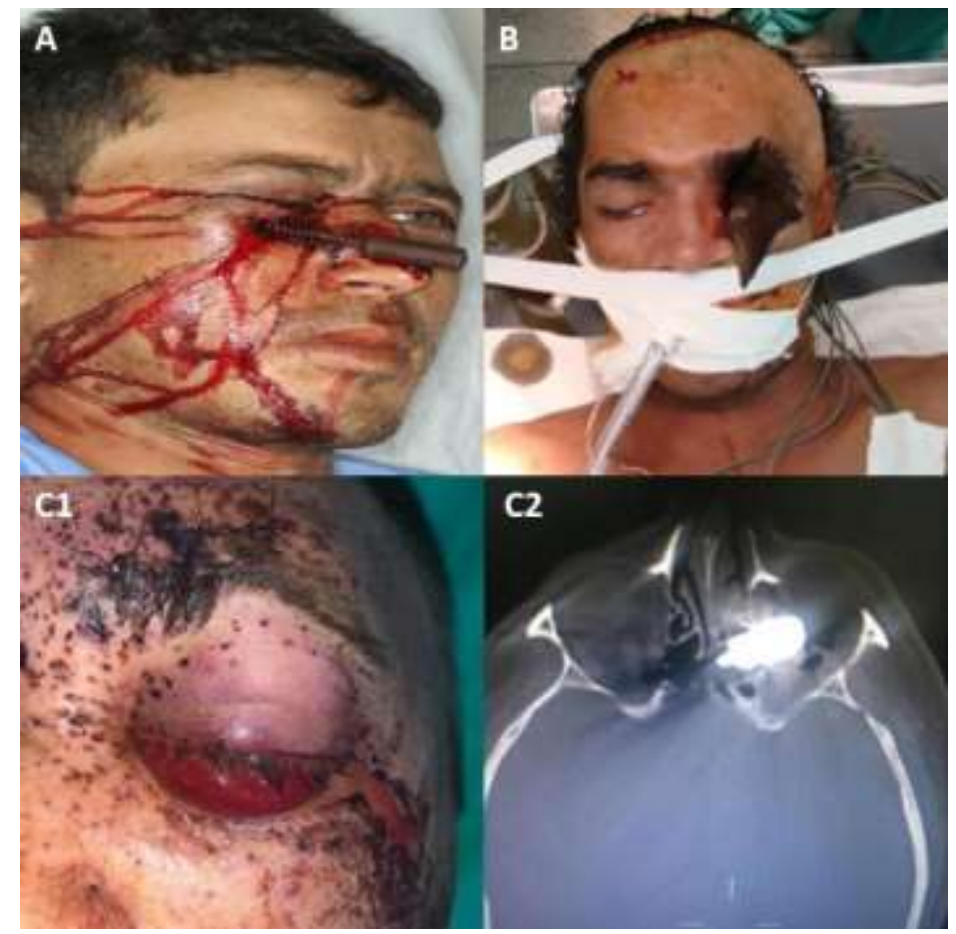

Fonte: Autores.

Apenas uma paciente apresentou lesão por vidro (caso 10). Admitida na urgência após acidente com estilhaços de vidro, os quais penetraram na órbita esquerda, sendo realizada tomografia computadorizada, a qual demonstrou material de aspecto hipodenso. Após intervenção cirúrgica para retirada do objeto, paciente evoluiu sem sequelas.

A Tabela 1 mostra os dados coletados de doze pacientes. A idade média foi de 31 anos, sendo 91,6\% dos pacientes do sexo masculino. A etiologia dos casos em $50 \%$ dos casos foi por material de madeira, em 41,7\% foi por metal e em $8,3 \%$ por vidro. A tomografia computadorizada foi utilizada em todos os fornecendo resultados altamente efetivos, com precisão quanto à localização, tamanho e lesão nos tecidos adjacentes. Em 58,3\% dos casos ocorreram na órbita esquerda. A remoção cirúrgica foi a conduta realizada em todos os casos. Em todos os casos realizou-se reforço antitetânico, com vacina anti-tetânica, antibiótico (ceftrixona EV) e nos casos em que a madeira foi o corpo estranho, utilizou-se o fluconazol como anti-fúngico.

\subsection{Revisão de literatura e discussão}

Em 27,7\% dos artigos coletados, o corpo estranho era madeira, o que difere dos nossos casos, em que a madeira foi o CE predominante. Em cinco dos catorze artigos (tabela 2) acerca de lesões orbitocranianas por corpos estranhos, selecionados como exemplares de casos de corpos estranhos ilustram-se a conduta diagnóstica e terapêutica (Scofield-Kaplan et al., 2019; Garg et al., 2005; Bater et al., 2007; Wilson et al., 2016; Farhadi et al., 2009; Santos et al., 2010). Em todos os relatos de caso, foi realizada antibioticoterapia e remoção cirúrgica. Apenas em um dos artigos foi possível a recuperação da acuidade visual. Quanto aos exames de imagem escolhidos para o diagnóstico, em todos a tomografia computadorizada foi determinante e efetiva para detecção da trajetória, tamanho e relação com o tecido orbital adjacente. Wilson et al. 2016, além de utilizarem a tomografia computadorizada, apresentaram resultados ainda mais precisos obtidos com a ressonância magnética, devido ao fato da aparência da madeira ser semelhante à do tecido mole, o que dificulta a detecção. 
Tabela 2 - Levantamento bibliográfico acerca do manejo de corpos estranhos em órbita.

\begin{tabular}{|c|c|c|c|c|c|}
\hline Autor & Tipo de estudo & Corpo estranho & Exame de imagem & Tratamento & Desfecho \\
\hline $\begin{array}{l}\text { Scofield-Kaplan } \\
\text { et al., } 2018\end{array}$ & Relato de caso & Madeira & $\begin{array}{l}\text { Tomografia } \\
\text { computadorizada } \\
\text { com contraste }\end{array}$ & $\begin{array}{l}\text { Amoxicilina com clavulonato, } \\
\text { ampicilina intravenosa, } \\
\text { clindamicina e remoção cirúrgica } \\
\text { (orbitotomia anterior direita) }\end{array}$ & $\begin{array}{l}\text { Recuperação da } \\
\text { acuidade visual }\end{array}$ \\
\hline $\begin{array}{l}\text { Bater et al., } \\
\quad 2009\end{array}$ & Relato de caso & Madeira & $\begin{array}{l}\text { Tomografia } \\
\text { computadorizada }\end{array}$ & $\begin{array}{l}\text { Antibioticoterapia e remoção } \\
\text { cirúrgica (orbitotomia inferior) }\end{array}$ & $\begin{array}{c}\text { Sensação } \\
\text { infraorbital } \\
\text { normalizada }\end{array}$ \\
\hline $\begin{array}{l}\text { Garg et al., } \\
2004\end{array}$ & Relato de caso & $\begin{array}{l}\text { Fragmento ósseo } \\
\text { em órbita }\end{array}$ & $\begin{array}{l}\text { Fundoscopia, } \\
\text { Tomografia } \\
\text { computadorizada e } \\
\text { ultrassonografia }\end{array}$ & Nenhum tratamento foi realizado & $\begin{array}{l}\text { Acuidade visual, } \\
\text { pressão intraocular } \\
\text { e esclera restaurada }\end{array}$ \\
\hline $\begin{array}{l}\text { Assoumane et } \\
\text { al., } 2019\end{array}$ & Relato de caso & Vidro & $\begin{array}{l}\text { Tomografia } \\
\text { computadorizada e } \\
\text { angiotomografia } \\
\text { computadorizada }\end{array}$ & $\begin{array}{l}\text { Toxóide antitetânico, } \\
\text { antibioticoterapia e remoção } \\
\text { cirúrgica (craniotomia } \\
\text { frontotemporal direita) }\end{array}$ & Perda da visão \\
\hline Cho et al., 2017 & Relato de caso & Lápis de grafite & $\begin{array}{c}\text { Tomografia } \\
\text { computadorizada e } \\
\text { angiografia }\end{array}$ & $\begin{array}{c}\text { Antibioticoterapia e remoção } \\
\text { cirúrgica }\end{array}$ & Perda da visão \\
\hline $\begin{array}{l}\text { Elyadari et al., } \\
2018\end{array}$ & Relato de caso & $\begin{array}{l}\text { Arma branca } \\
\quad(\text { metal })\end{array}$ & $\begin{array}{l}\text { Radiografia, } \\
\text { tomografia coronal } \\
\text { e angiografia }\end{array}$ & $\begin{array}{c}\text { Antibioticoterapia e remoção } \\
\text { cirúrgica }\end{array}$ & $\begin{array}{c}\text { Edema palpebral e } \\
\text { quemose } \\
\text { hemorrágica }\end{array}$ \\
\hline $\begin{array}{l}\text { De Morais et } \\
\text { al., } 2015\end{array}$ & Relato de caso & $\begin{array}{l}\text { Culatra de arma } \\
\quad(\text { metal })\end{array}$ & $\begin{array}{l}\text { Tomografia } \\
\text { computadorizada } \\
\text { tridimensional e } \\
\text { biomicroscopia }\end{array}$ & $\begin{array}{l}\text { Antibióticos de amplo espectro, } \\
\text { remoção cirúrgica (acesso } \\
\text { temporal) e toxóide antitetânico }\end{array}$ & $\begin{array}{l}\text { Perda da acuidade } \\
\text { visual e ausência de } \\
\text { reflexos pupilares }\end{array}$ \\
\hline $\begin{array}{l}\text { Okay et al., } \\
2009\end{array}$ & Relato de caso & $\begin{array}{l}\text { Arma branca } \\
\quad(\text { metal })\end{array}$ & $\begin{array}{c}\text { Radiografia e } \\
\text { tomografia } \\
\text { computadorizada }\end{array}$ & $\begin{array}{l}\text { Antibióticos de amplo espectro, } \\
\text { remoção cirúrgica (acesso } \\
\text { temporal) e toxóide antitetânico }\end{array}$ & $\begin{array}{c}\text { Perda visual total e } \\
\text { ausência de reflexos } \\
\text { pupilares em olho } \\
\text { direito. }\end{array}$ \\
\hline $\begin{array}{l}\text { Tabibkhooei et } \\
\text { al., } 2019\end{array}$ & Relato de caso & $\begin{array}{l}\text { Arma branca } \\
\text { (metal) }\end{array}$ & $\begin{array}{l}\text { Tomografia } \\
\text { computadorizada e } \\
\text { angiotomografia } \\
\text { computadorizada }\end{array}$ & $\begin{array}{l}\text { Antibióticos de amplo espectro e } \\
\text { remoção cirúrgica }\end{array}$ & $\begin{array}{l}\text { Recuperação } \\
\text { completa }\end{array}$ \\
\hline $\begin{array}{l}\text { Farhadi et al., } \\
2009\end{array}$ & Relato de caso & Plástico & $\begin{array}{c}\text { Tomografia } \\
\text { computadorizada }\end{array}$ & $\begin{array}{c}\text { Antibioticoterapia e remoção } \\
\text { cirúrgica }\end{array}$ & $\begin{array}{l}\text { Permaneceu } \\
\text { severamente } \\
\text { incapacitada }\end{array}$ \\
\hline $\begin{array}{l}\text { Wilson et al., } \\
2016\end{array}$ & Relato de caso & Madeira & $\begin{array}{l}\text { Tomografia } \\
\text { computadorizada e } \\
\text { ressonância } \\
\text { magnética }\end{array}$ & $\begin{array}{c}\text { Antibioticoterapia e remoção } \\
\text { cirúrgica }\end{array}$ & Sem informações \\
\hline $\begin{array}{l}\text { Santos et al., } \\
2010\end{array}$ & Série de casos & Madeira & $\begin{array}{l}\text { Radiografia e } \\
\text { tomografia } \\
\text { computadorizada }\end{array}$ & $\begin{array}{c}\text { Antibioticoterapia e remoção } \\
\text { cirúrgica }\end{array}$ & Sem informações \\
\hline
\end{tabular}


Fonte: Autores.

Em 8.3\% dos artigos coletados, o material relatado foi vidro (Srivastava et al., 2012; Assoumane et al., 2019; ScofieldKaplan et al., 2019). A tomografia computadorizada determinou a trajetória do objeto, pois a radiografia não seria suficiente e a angiotomografia computadorizada foi realizada para identificar as lesões vasculares presentes. O tratamento consistiu em toxóide antitetânico, em virtude do desconhecimento da situação vacinal, antibioticoterapia e remoção cirúrgica.

Em um caso, foi relatado osso do assoalho orbitário como "corpo estranho" (Garg et al., 2005). O diagnóstico foi fundamentado na realização de fundoscopia, tomografia computadorizada e ultrassonografia, precisos na identificação do fragmento ósseo penetrando na esclera, coróide e retina. Nenhum tratamento foi realizado, três meses depois, nenhuma queixa ou complicação foi relatada, sendo realizado nesse caso tratamento conservador.

Em um único artigo, material de plástico foi o corpo estranho penetrante (Farhadi et al., 2009. A tomografia computadorizada foi suficiente para determinar a conduta, que consistiu em antibioticoterapia e remoção cirúrgica.

Em 33,3\%, corpos metálicos caracterizaram a etiologia do trauma. Foi imprescindível a utilização de tomografia computadorizada, em dois casos complementada com uso de contraste, em dois realizados com reconstrução tridimensional. Angiografia e biomicroscopia foram menos utilizados, constando respectivamente apenas um caso cada, dos abordados.

\section{Considerações Finais}

Lesões penetrantes em órbita e a detecção tardia de fragmentos retidos podem resultar em perda da visão e até óbito. Os exames de imagem adequados são essenciais para a detecção desses corpos retidos. Recomenda-se na literatura que a detecção e remoção dos corpos estranhos ocorra em até 48 horas, prescrição de antibioticoterapia e antimicrobianos. A tomografia computadorizada, a ressonância magnética e a angiografia por tomografia computadorizada são modalidades de imagem frequentemente eficazes na determinação do trajeto do objeto, estruturas anatômicas lesadas, extensão das lesões, e possíveis comprometimentos neurovasculares. A exploração cirúrgica por uma equipe multidisciplinar é essencial.

\section{Financiamento}

Apoio financeiro: não houve financiamento.

\section{Conflitos de interesses}

Não houveram conflitos de interesses dos pesquisadores.

\section{Referências}

Albert, D. M., Burns, W. P., \& Scheie, H. G. (1985). Severe orbitocranial foreign-body injury. American journal of ophthalmology, 60(6), $1109-1111$.

Assoumane, I.; Mahdia, T.; Morsli, A (2019). An orbitocranial glass foreign body in contact of skull base great vessels following a physical aggression. Visual Journal Of Emergency Medicine, 14 (1), 59-60.

Balasubramanian, C., Kaliaperumal, C., Jadun, C. K., \& Dias, P. S. (2009). Transorbital intracranial penetrating injury-an anatomical classification. Surgical neurology, 71(2), 238-240.

Baranwal, V. K., Gupta, R. P., Johri, S., Dutta, A. K., Murthy, P. K., \& Avinash Mishra (2016). A case of orbitocranial foreign body. Medical journal, Armed Forces India, 72(1), 82-84.

Bater, M. C., Scott, R., \& Flood, T. R. (2007). Use of an inferior orbitotomy for safe removal of a wooden foreign body penetrating the orbit. British Journal of Oral and Maxillofacial Surgery. 45(8), 664-6.

Cho, W. K., Ko, A. C., Eatamadi, H., Al-Ali, A., Abboud, J. P., Kikkawa, D. O., \& Korn, B. S. (2017). Orbital and Orbitocranial Trauma From Pencil Fragments: Role of Timely Diagnosis and Management. American journal of ophthalmology, 180, 46-54. 
Chowdhury, F. H., Haque, M. R., Hossain, Z., Chowdhury, N. K., Alam, S. M., \& Sarker, M. H. (2016). Nonmissile Penetrating Injury to the Head: Experience with 17 Cases. World neurosurgery, 94, 529-543.

de Morais, H. H., Barbalho, J. C., de Souza Dias, T. G., et al (2015). Temporal Approach to Removal of a Large Orbital Foreign Body. Craniomaxillofac Trauma Reconstr. 8(3):234-238.

Del Verme, J., Giordan, E., Marton, E., Zanata, R., Di Paola, F., Canova, G., \& Longatti, P. (2020). Classification of orbitocranial wooden foreign body penetration injuries: what to do when they violate the intracranial space? A systematic review. Journal of neurosurgical sciences, 64(2), 190-199.

Di Gaeta, A., Giurazza, F., Capobianco, E., Diano, A., \& Muto, M. (2017). Intraorbital wooden foreign body detected by computed tomography and magnetic resonance imaging. The neuroradiology journal, 30(1), 88-91.

Elyadari, M., Bouchama, H., Harchali, S., et al (2018). Orbital metallic foreign body: Case report. J Fr Ophtalmol. 41(5), 205-206.

Farhadi, M. R., Becker, M., Stippich, C., et al (2009). Transorbital penetrating head injury by a toilet brush handle. Acta Neurochir (Wien). 151(6), 685-7.

Fulcher, T. P., McNab, A. A., \& Sullivan, T. J. (2002). Clinical features and management of intraorbital foreign bodies. Ophthalmology, 109(3), 494-500.

Garg, S. J., Benson, W., Fineman, M., et al (2005). Bone from an orbital floor fracture causing an intraocular foreign body. Am J Ophthalmol. 139(3), 543-5.

Gönül, E., Erdoğan, E., Taşar, M., Yetişer, S., Akay, K. M., Düz, B., Bedük, A., \& Timurkaynak, E. Penetrating orbitocranial gunshot injuries. Surg Neurol. 2005

63(1):24-30.

Hada, M., Chandra, P., \& Gangwe, A. (2016). An unusual case of protruding retro-orbital metallic foreign body. Indian journal of ophthalmology, 64(8), 604606.

Joos, Z. P., Longenecker, L. G., \& Patel, B. C. (2016). Orbital foreign body masquerading as orbital air. Clinical \& experimental ophthalmology, 44(7), 637639.

Kane, N., Jamjoom, A., \& Teimory, M. (1991). Penetrating orbitocranial injury. Injury, 22(4), 326-327.

McNab, A. A., \& Satchi, K. (2012). Orbital Foreign Bodies and Penetrating Orbital Injuries. In: Black E., Nesi F., Calvano C., Gladstone G., Levine M. (eds) Smith and Nesi’s Ophthalmic Plastic and Reconstructive Surgery. Springer, New York, NY.

Mzimbiri, J. M., Li, J., Bajawi, M. A., Lan, S., Chen, F., \& Liu, J. (2016). Orbitocranial Low-Velocity Penetrating Injury: A Personal Experience, Case Series, Review of the literature, and Proposed Management Plan. World neurosurgery, 87, 26-34.

Nasr, A. M., Haik, B. G., Fleming, J. C., Al-Hussain, H. M., \& Karcioglu, Z. A. (1999). Penetrating orbital injury with organic foreign bodies. Ophthalmology, 106(3), 523-532.

Okay, O., Dağlioğlu, E., Ozdol, C., Uckun, O., Dalgic, A., \& Ergungor, F. (2009). Orbitocerebral injury by a knife: case report. Neurocirugia (Asturias, Spain), 20(5), 467-469.

Park, S. J., \& Chi, M. (2018). Transorbital Penetrating Intracranial Injury by a Battery. The Journal of craniofacial surgery, 29(1), e61-e64.

Santos, T. S., Melo, A. R., Moraes, H. H. A., et al (2010). Impacted foreign bodies in orbital region: review of nine cases. Arq. Bras. Oftalmol. 73(5), 438-442.

Scofield-Kaplan, S. M., Weidman, E. K., Moonis, G., et al (2019). Orbital wooden foreign body manifesting as hyperdensity on computed tomography. $J$ aapos. 23(1), 45-7.

Shukla, B. (2016). New classification of ocular foreign bodies. Chinese journal of traumatology = Zhonghua chuang shang za zhi, 19(6), 319-321.

Srivastava, C., Singh, S. K., Ojha, B. K, et al. (2012). Intracranial penetrating glass injury: A case report. The Indian Journal of Neurotrauma, 9(1), 64-66.

Tabibkhooei, A., Aslaninia, A., \& Anousha, K. (2019). Childhood Transorbital Skull Base Penetrating Injury: Report of 2 Cases and Review of Literature. World Neurosurgery. 131(1), 213-6.

Wilson, M. H., Collins, T. R., \& Revington, P. J. (2016). Orbitocranial wooden foreign body retrieved by transcranial and superior orbitotomy. The British journal of oral \& maxillofacial surgery, 54(9), 1050-1051. 